\title{
Robust guaranteed-cost preview repetitive control for polytopic uncertain discrete-time systems ${ }^{\text {th }}$
}

\author{
Yong-Hong Lan, Jun-Jun Xia \\ School of Information Engineering, Xiangtan University, Xiangtan, Hunan 411105, \\ China.
}

\begin{abstract}
A robust guaranteed cost preview repetitive controller is proposed for a class of polytopic uncertain discrete-time systems. In order to improve the tracking performance, the repetitive controller combined with preview compensator is inserted in the forward channel. By using the $L$-order forward difference operator, an augmented dynamic system is constructed. Then, the guaranteed cost preview repetitive control problem is transformed into the guaranteed cost control problem for the augmented dynamic system. For given performance index, the sufficient condition of asymptotic stability for the closed-loop system is derived by combining parameter-dependent Lyapunov function method with linear matrix inequality (LMI) techniques. By incorporating the controller obtained into the original system, the guaranteed-cost preview repetitive controller is derived. A numerical example is also included to show the effectiveness of the proposed method.
\end{abstract}

Keywords: robust control; preview control; repetitive control; controller design; uncertain systems

\section{Introduction}

In some control systems, it is hoped that the output can track reference signal without steady-state error, even in the presence of uncertainty and/or exogenous disturbances. Preview control $[1,2]$ is one of the many ways for

\footnotetext{
* This work was supported in part by the National Natural Science Foundation of China (61573298) and Scientific Research Fund of Hunan Provincial Education Department (15B238).
} 
solve this kind of control problem. The basic idea of preview control is to use known future information of the reference or disturbance signals. It was found that preview control can enhance performance of the closed-loop system [3]. In recent years, the problem of preview control has received considerable attention and various control structures and algorithms have been proposed $[4,5,6,7]$.

As a typical regulator method, the linear quadratic regulator (LQR) optimal control is a powerful technique for designing preview controller $[6,7,8]$. In [6], an optimal preview control was investigated for discrete-time Markovian jump linear systems. The optimal semi-active preview control of a car vehicle model was considered in [7], in which the optimal control with preview minimizes a performance index by solving a multi-objective optimization problem. In [8], to track triangular reference, the LQR optimal control with preview compensation for a dual-stage actuator system was proposed. In [9], the constrained optimal preview control of dual-stage actuators was proposed and the problem was solved in terms of quadratic programming providing an optimal solution with respect to a quadratic criterion. In [10], by using the reference and disturbance signal's preview information to calculate the control input, an optimal feed-forward preview controller was presented for single-input single-output systems. In order to estimate the unmeasurable state vector, a state observer-based optimal preview controller was developed in [11]. Other relevant work may be referred to [12]. As well known, the LQRbased optimal preview control can not be applied to deal with the uncertain systems.

For uncertain systems, it will become difficult to design preview controller. In [13], a robust tracking problem for a polytopic uncertain system subject to a previewable reference signal was considered. The developed design method in terms of linear matrix inequalities (LMIs) achieved good robust tracking performance. In [14], an LMI-based solution to the reliable robust preview tracking problems against actuator faults for discrete LTI systems with polytopic uncertainties was provided. In [15], the problem of multi-model adaptive preview control was proposed for discrete-time systems with unknown piecewise constant coefficients. In [16], the author overviewed the preview control research achievement and discussed the main problems. Very recently, for a class of polytopic uncertain discrete systems, by combining parameter-dependent Lyapunov function method with LMI techniques, the problem of static output feedback preview tracking control was presented in [17]. For a class of uncertain discrete-time systems, an LMI-based preview 
controller was proposed in [18]. The proposed method can be extended completely to other time varying systems for preview control.

Repetitive control (RC), which was first developed in the early 1980s [19], is a very useful strategy for tracking periodic reference signals [20, 21, 22]. Although repetitive control method was originally proposed in continuoustime systems, it was realized by digital computer in the actual applications. The direct design of repetitive controller in discrete time domain is more practical. In [23], a discrete-time variable-structure repetitive control was proposed. The presented control scheme ensures robust stability of the resultant quasi-sliding-mode dynamics to parameter variations and exogenous disturbances. The authors in [24] have investigated a positive realness problem raised in the discrete repetitive control. The sufficient condition of the repetitive control stability can be satisfied through constructing a discrete positive real transfer function. Based on two-dimensional system theory, the problem of designing discrete-time repetitive-control system were studied in $[25,26]$. By the same argument, the observer-based repetitive controller design method for discrete-time systems with uncertainties was presented in [27].

On the other hand, for many control system, when a system is dependent on uncertain parameters, it is desirable to design a control system is not only asymptotically stable but also guarantees an adequate level of control performance. This kind of control problem, is called guaranteed-cost control [28, 29]. For a class of uncertain discrete-time systems, a guaranteed cost preview controller was proposed in [18]. For discrete-time linear systems with uncertainties, a method of designing a guaranteed cost robust discrete repetitive controller based on two-dimensional model was proposed in [30]. For continuous-time linear systems, in [31], the design of non-fragile guaranteed-cost repetitive control was studied. It should be noted that the guaranteed-cost preview repetitive control problems have not been considered so far.

In this paper, motivated by the above results, a new configuration of repetitive control system with preview feedforward compensation, as well as the guaranteed-cost preview repetitive controller design method is presented. Using the lift technique, an augmented state-space dynamic system is constructed. The robust guaranteed-cost preview repetitive controller design problem is then transformed into the guaranteed-cost control problem of the augmented system. By combining parameter-dependent Lyapunov function method with LMI techniques, the guaranteed-cost preview repetitive con- 
troller is obtained.

The rest of this paper is organized as follows. Section 2 presents problem formulation and preliminaries. Section 3 describes the construction of an augmented dynamic system. The design of guaranteed-cost preview repetitive controller is explained in Section 4. Section 5 presents a numerical example that demonstrates the effectiveness of the method. Finally, Section 6 gives some concluding remarks.

Throughout this paper, $\mathbb{R}^{n}$ denotes an $n$-dimensional Euclidean space, $\mathbb{R}^{n \times m}$ is the set of all $n \times m$ real matrices, $I$ means an identity matrix of appropriate order, $0_{p \times p}$ is the $p \times p$ zero matrix (the subscript is omitted if the dimension is clear.), and $*$ indicates the entries below the main diagonal of a symmetric block matrix. The notation $X>0(<0)$ means that matrix $X$ is positive (negative) definite.

\section{Problem Formulation and Preliminaries}

Consider the following uncertain SISO discrete-time system:

$$
\left\{\begin{array}{l}
x(k+1)=A(\theta) x(k)+B(\theta) u(k)+E d(k), \\
y(k)=C x(k) .
\end{array}\right.
$$

where $x(k) \in \mathbb{R}^{n}$ is the state, $u(k) \in \mathbb{R}^{m}$ is the control input, $d(k) \in \mathbb{R}^{p}$ is a periodic disturbance, and $y(k) \in \mathbb{R}^{q}$ is the output of the plant. $E, C$ are constant matrices with appropriate dimensions. $A(\theta), B(\theta)$ are uncertain matrices with appropriate dimensions and are given by

$$
\left[\begin{array}{ll}
A(\theta) & B(\theta)
\end{array}\right]=\sum_{i=1}^{s} \theta_{i}\left[\begin{array}{ll}
A_{i} & B_{i}
\end{array}\right]
$$

where $A_{i}, B_{i}(i=1,2, \ldots, s)$ are constant matrices with appropriate dimensions, and $\theta=\left[\theta_{1}, \theta_{2}, \cdots, \theta_{s}\right]^{T}$ is the uncertain constant parameter vector satisfying

$$
\theta \in \Theta:=\left\{\theta \in \mathbb{R}^{s} \mid \theta_{i} \geq 0,(i=1,2, \ldots, s), \sum_{i=1}^{s} \theta_{i}=1\right\} .
$$

Let $r(k) \in \mathbb{R}^{q}$ be a periodic reference signal to be tracked by $y(k) \in \mathbb{R}^{q}$ and

$$
e(k)=r(k)-y(k)
$$


be the tracking error.

Throughout this paper, two assumptions are made:

A1: The reference signal $r(k)$ and disturbance signal $d(k)$ are periodic and both of the period are $L$.

A2: The preview length of the reference signal $r(k)$ and disturbance signal $d(k)$ is $M_{r}$ and $M_{d}\left(M_{r}, M_{d}\right.$ are less than $\left.L\right)$, respectively. That is, at each time $k$, the $M_{r}\left(M_{d}\right)$ future values, $r(k+1), r(k+2), \ldots, r\left(k+M_{r}\right)$ $\left(d(k+1), d(k+2), \ldots, d\left(k+M_{d}\right)\right)$ as well as the present and past values of the reference signal are available.

Remark 2.1. In fact, the disturbance signal and the reference signal are not necessarily the same period. In this case, the period of the repetitive controller can be set as the minimum common multiple of the period of reference signal and interference signal.

Remark 2.2. Clearly, a conventional feedback control system does not take full advantage of the known future values, i.e., $M_{r}=0$ and $M_{d}=0 . H y$ pothes A2 indicates that the reference signal and the disturbance signals have impacted performance of the control system significantly only for a certain time period [17, 32, 33].

The basic configuration of a discrete-time repetitive control system [34] is shown in Fig. 1, where $P(z)$ is the compensated plant, $r(k)$ is a periodic reference input with period $L$, and $C_{R}(z)$ is a repetitive controller. The

Figure 1: Basic discrete-time repetitive-control system.

output of the repetitive controller, $v(k)$, is

$$
\begin{gathered}
C_{R}(z)=\frac{1}{1-z^{-L}} \\
v(k)= \begin{cases}v(k-L)+e(k), & k \geq L, \\
e(k), & k<L .\end{cases}
\end{gathered}
$$


Figure 2: Configuration of repetitive-control system with preview compensation.

This paper concerns the repetitive-control system configuration in Fig. 2 , where $G(z)$ is the controlled plant as described in system $(1) . K_{r}(j)(j=$ $\left.0,1,2, \ldots, M_{r}\right)$ and $K_{d}(j)\left(j=0,1,2, \ldots, M_{d}\right)$ are the gains of the reference signal and disturbance signal preview compensator, respectively. $K_{e}(j)(j=$ $0,1,2, \ldots, L-1)$ and $K_{y}$ are the gains of the repetitive controller and the output feedback controller.

The objective is to design a guaranteed-cost preview control law with the form of

$$
u(k)=\sum_{j=0}^{L-1} K_{e}(j) v(k-j)+K_{y} y(k)+\sum_{j=0}^{M_{r}} K_{r}(j) r(k+j)+\sum_{j=0}^{M_{d}} K_{d}(j) d(k+j) .
$$

Remark 2.3. The preview repetitive controller (7) consists of four parts: the first part is repetitive control, the second term represents output feedback, the third and the fourth represent the preview action based on the future value of the previewable reference signal and the disturbance signal. Besides, considering that the system states are not measurable, the static output feedback is used instead of taking the state feedback.

For uncertain discrete-time system (1), we introduce a quadratic cost function

$$
J=\sum_{k=0}^{\infty}\left[e(k)^{T} Q_{e} e(k)+\Delta u(k)^{T} R \Delta u(k)\right]
$$

where $\Delta u(k)=u(k)-u(k-L), Q_{e}$ and $R$ are the given symmetric positive definite weighted matrices.

A guaranteed-cost preview controller is defined as follows. 
Definition 2.4. For the uncertain discrete-time system (1) and the cost function (8), if there exist a preview repetitive-control law (7) that produces a control input $u^{*}(k)$, and a positive constant $J^{*}$ such that $\lim _{k \rightarrow \infty} e(k)=0$, and for all admissible uncertainties the closed-loop system is robustly stable and the value of the cost function (8) satisfies $J \leq J^{*}$, then $J^{*}$ is said to be a guaranteed cost; and $u^{*}(k)$ is said to be a guaranteed-cost preview repetitive-controller for the cost function (8).

To obtain our main results, the following lemmas will be used.

Lemma 2.5. [35] For appropriately dimensioned matrices $T, S, R$ and $N$, and scalar $\beta, T+S^{T} R^{T}+R S<0$ if the following condition holds:

$$
\left[\begin{array}{cc}
T & \left(\beta R^{T}+N S^{T}\right)^{T} \\
\beta R^{T}+N S^{T} & -\beta N-\beta N^{T}
\end{array}\right]<0 .
$$

Lemma 2.6. [36] For the matrices $L \in \mathbb{R}^{S \times S}, H \in \mathbb{R}^{S \times S}$ and $H>0$, one has

$$
-L^{T} H^{-1} L \leq-L-L^{T}+H
$$

\section{Construction of Augmented Dynamic System}

In this section, by using the $L$-order forward difference operator, an augmented state-space dynamic system is first presented, which contains previewed information, error vectors and states of the system. Then, the guaranteed cost preview repetitive control problem is transformed into the guaranteed cost control problem for the augmented dynamic system.

For all the system variables, define the $L$-order forward difference operator as

$$
\Delta \xi(k)=\xi(k)-\xi(k-L), \xi \in\{x, y, u, r, d\} .
$$

Taking the $L$-order difference operator on both sides of the state equation (1) and error equation (4), we derive

$$
\left\{\begin{array}{c}
e(k+1)=e(k+1-L)+\Delta r(k+1)-C A(\theta) \Delta x(k) \\
-C B(\theta) \Delta u(k)-C E \Delta d(k), \\
\Delta x(k+1)=A(\theta) \Delta x(k)+B(\theta) \Delta u(k)+E \Delta d(k), \\
\Delta y(k)=C \Delta x(k) .
\end{array}\right.
$$




\section{Defining}

$$
\left\{\begin{array}{l}
x_{e}(k)=\left[\begin{array}{ll}
e^{T}(k) & e^{T}(k-1) \\
\bar{x}(k)=\left[x_{e}^{T}(k)\right. & \Delta x^{T}(k)
\end{array}\right]^{T},
\end{array}\right.
$$

and combining (12) and (13), we have

$$
\bar{x}(k+1)=\bar{A}(\theta) \bar{x}(k)+\bar{B}(\theta) \Delta u(k)+G_{r} \Delta r(k+1)+G_{d} \Delta d(k),
$$

where

$$
\begin{aligned}
& \bar{A}(\theta)=\left(\begin{array}{ccccc}
0 & \cdots & 0 & I & -C A(\theta) \\
I & \cdots & 0 & 0 & 0 \\
\vdots & & \vdots & \vdots & \vdots \\
0 & \cdots & I & 0 & 0 \\
0 & \cdots & 0 & 0 & A(\theta)
\end{array}\right), \\
& \bar{B}(\theta)=\left(\begin{array}{c}
-C B(\theta) \\
0 \\
\vdots \\
0 \\
B(\theta)
\end{array}\right), G_{r}=\left(\begin{array}{c}
I \\
0 \\
\vdots \\
0 \\
0
\end{array}\right), G_{d}=\left(\begin{array}{c}
-C E \\
0 \\
\vdots \\
0 \\
E
\end{array}\right) .
\end{aligned}
$$

From (2), the uncertain matrices $\bar{A}(\theta)$ and $\bar{B}(\theta)$ can be rewritten as

$$
\begin{aligned}
\bar{A}(\theta) & =\left(\begin{array}{ccccc}
0 & \cdots & 0 & I & -C \sum_{i=1}^{s} \theta_{i} A_{i} \\
I & \cdots & 0 & 0 & 0 \\
\vdots & & \vdots & \vdots & \vdots \\
0 & \cdots & I & 0 & 0 \\
0 & \cdots & 0 & 0 & \sum_{i=1}^{s} \theta_{i} A_{i}
\end{array}\right) \\
& =\sum_{i=1}^{s} \theta_{i}\left(\begin{array}{ccccc}
0 & \cdots & 0 & I & -C A_{i} \\
I & \cdots & 0 & 0 & 0 \\
\vdots & & \vdots & \vdots & \vdots \\
0 & \cdots & I & 0 & 0 \\
0 & \cdots & 0 & 0 & A_{i}
\end{array}\right)=\sum_{i=1}^{s} \theta_{i} \bar{A}_{i},
\end{aligned}
$$




$$
\bar{B}(\theta)=\left(\begin{array}{c}
-C \sum_{i=1}^{s} \theta_{i} B_{i} \\
0 \\
\vdots \\
0 \\
\sum_{i=1}^{s} \theta_{i} B_{i}
\end{array}\right)=\sum_{i=1}^{s} \theta_{i}\left(\begin{array}{c}
-C B_{i} \\
0 \\
\vdots \\
0 \\
B_{i}
\end{array}\right)=\sum_{i=1}^{s} \theta_{i} \bar{B}_{i}
$$

For uncertain system (14), we specify the observation equation as

$$
e(k)=\bar{C} \bar{x}(k)
$$

where $\bar{C}=\left[\begin{array}{ll}I_{q} & 0_{(L-1) q+n}\end{array}\right]$.

Defining

$$
X_{r}(k)=\left(\begin{array}{c}
\Delta r(k) \\
\Delta r(k+1) \\
\vdots \\
\Delta r\left(k+M_{r}\right)
\end{array}\right), \quad X_{d}(k)=\left(\begin{array}{c}
\Delta r(k) \\
\Delta r(k+1) \\
\vdots \\
\Delta r\left(k+M_{d}\right)
\end{array}\right) \text {, }
$$

it follows from the assumptions $\mathrm{A} 1$ and $\mathrm{A} 2$ that $X_{r}(k), X_{d}(k)$ satisfy

$$
\begin{gathered}
X_{r}(k+1)=A_{r} X_{r}(k), \\
X_{d}(k+1)=A_{d} X_{d}(k),
\end{gathered}
$$

where $A_{r} \in \mathbb{R}^{q\left(M_{r}+1\right) \times q\left(M_{r}+1\right)}, A_{d} \in \mathbb{R}^{p\left(M_{d}+1\right) \times p\left(M_{d}+1\right)}$, and

$$
A_{r}=\left(\begin{array}{ccccc}
0 & I & 0 & \cdots & 0 \\
0 & \ddots & \ddots & \ddots & \vdots \\
\vdots & & \ddots & \ddots & 0 \\
0 & \cdots & \cdots & 0 & I \\
0 & \cdots & \cdots & 0 & 0
\end{array}\right), A_{d}=\left(\begin{array}{ccccc}
0 & I & 0 & \cdots & 0 \\
0 & \ddots & \ddots & \ddots & \vdots \\
\vdots & & \ddots & \ddots & 0 \\
0 & \cdots & \cdots & 0 & I \\
0 & \cdots & \cdots & 0 & 0
\end{array}\right)
$$

Now defining the augmented state vector

$$
\widehat{x}(k)=\left(\begin{array}{c}
\bar{x}(k) \\
X_{r}(k) \\
X_{d}(k)
\end{array}\right),
$$


from (14), (19) and (20), we can obtain the augmented system

$$
\widehat{x}(k+1)=\widehat{A}(\theta) \widehat{x}(k)+\widehat{B}(\theta) \Delta u(k),
$$

where

$$
\begin{aligned}
& \widehat{A}(\theta)=\left(\begin{array}{ccc}
\sum_{i=1}^{s} \theta_{i} \bar{A}_{i} & G_{x r} & G_{x d} \\
0 & A_{r} & 0 \\
0 & 0 & A_{d}
\end{array}\right)=\sum_{i=1}^{s} \theta_{i}\left(\begin{array}{ccc}
\bar{A}_{i} & G_{x r} & G_{x d} \\
0 & A_{r} & 0 \\
0 & 0 & A_{d}
\end{array}\right)=\sum_{i=1}^{s} \theta_{i} \widehat{A}_{i}, \\
& \widehat{B}(\theta)=\left(\begin{array}{c}
\sum_{i=1}^{s} \theta_{i} \bar{B}_{i} \\
0 \\
0
\end{array}\right)=\sum_{i=1}^{s} \theta_{i}\left(\begin{array}{c}
\bar{B}_{i} \\
0 \\
0
\end{array}\right)=\sum_{i=1}^{s} \theta_{i} \widehat{B}_{i} \\
& G_{x r}=\left(\begin{array}{lllll}
0 & G_{r} & 0 & \cdots & 0
\end{array}\right), G_{x d}=\left(\begin{array}{lllll}
G_{d} & 0 & 0 & \cdots & 0
\end{array}\right) .
\end{aligned}
$$

For augmented system (22), the observation equation can be written as

$$
Z(k)=C_{Z} \widehat{x}(k),
$$

where

$$
C_{Z}=\left(\begin{array}{cccc}
I_{L} & & & \\
& C & & \\
& & I_{M_{r}+1} & \\
& & & I_{M_{d}+1}
\end{array}\right) .
$$

Therefore, by (22) and (26), one can get

$$
\left\{\begin{array}{l}
\widehat{x}(k+1)=\widehat{A}(\theta) \widehat{x}(k)+\widehat{B}(\theta) \Delta u(k), \\
Z(k)=C_{Z} \widehat{x}(k) .
\end{array}\right.
$$

In terms of the augmented state vector $\widehat{x}(k)$, the performance index (8) can be rewritten as

$$
\widehat{J}=\sum_{k=0}^{\infty}\left[\widehat{x}(k)^{T} Q_{\widehat{x}} \widehat{x}(k)+\Delta u(k)^{T} R \Delta u(k)\right],
$$

where $Q_{\hat{x}} \in R^{\left[L \times q+n+\left(M_{r}+1\right) \times q+\left(M_{d}+1\right) \times p\right] \times\left[L \times q+n+\left(M_{r}+1\right) \times q+\left(M_{d}+1\right) \times p\right]}$, and

$$
Q_{\hat{x}}=\left(\begin{array}{cccc}
Q_{e} & & & \\
& 0 & & \\
& & \ddots & \\
& & & 0
\end{array}\right),
$$

and $R$ is the same as in performance index (8). 


\section{Design of Guaranteed-cost Preview Repetitive Controller}

Note that the proposed preview repetitive controller (7) can be written as

$$
\begin{aligned}
\Delta u(k) & =\sum_{j=0}^{L-1} K_{e}(j) e(k-j)+K_{y} \Delta y(k) \\
& +\sum_{j=0}^{M_{r}} K_{r}(j) \Delta r(k+i)+\sum_{j=0}^{M_{d}} K_{d}(j) \Delta d(k+j) .
\end{aligned}
$$

Set

$$
\begin{aligned}
& K_{e}(j)=\sum_{i=1}^{s} \alpha_{i} K_{e i}(j), \quad K_{y}=\sum_{i=1}^{s} \alpha_{i} K_{y i}, \\
& K_{r}(j)=\sum_{i=1}^{s} \alpha_{i} K_{r i}(j), \quad K_{d}(j)=\sum_{i=1}^{s} \alpha_{i} K_{d i}(j),
\end{aligned}
$$

where $\alpha_{i}(i=1,2, \ldots, s)$ are adjustable variables, and $\alpha_{i} \geq 0, \sum_{i=1}^{s} \alpha_{i}=1$. Furthermore, denoting

$$
\begin{aligned}
K_{i}= & {\left[K_{e i}(0), K_{e i}(1), \cdots, K_{e i}(L-1), K_{y i},\right.} \\
& K_{r i}(0), K_{r i}(1), \cdots, K_{r i}\left(M_{r}\right), \\
& \left.K_{d i}(0), K_{d i}(1), \cdots, K_{d i}\left(M_{d}\right)\right],
\end{aligned}
$$

it follows from (29) to (31), we have

$$
\Delta u(k)=\left(\sum_{i=0}^{s} \alpha_{i} K_{i}\right) Z(k) .
$$

If we denote $\alpha=\left(\begin{array}{llll}\alpha_{1} & \alpha_{2} & \ldots & \alpha_{s}\end{array}\right)^{T} \in R^{s}$ and $K(\alpha)=\sum_{i=1}^{s} \alpha_{i} K_{i}$, then

$$
\Delta u(k)=K(\alpha) Z(k) .
$$

Therefore, it is obvious that if one can design a guaranteed cost static output feedback controller (33) to robustly stabilize the augmented system (27), then 
the preview repetitive controller (7) can also stabilize the original system (1) and such that $\lim _{k \rightarrow \infty} e(k)=0$.

In the following, we will give the sufficient conditions for the existence of a guaranteed cost static output feedback controller (33) to robustly stabilize the augmented system (27).

Theorem 4.1. For the augmented system (27) with the performance index (28), if there exist positive defined parameter-dependent matrix $P(\theta)$ and reversible matrix $G(\alpha)=\sum_{i=1}^{s} \alpha_{i} G_{i}$ such that

$$
\left(\begin{array}{ccc}
\Pi_{11} & * & * \\
{\left[\widehat{A}(\theta)+\widehat{B}(\theta) K(\alpha) C_{Z}\right] G(\alpha)} & -P(\theta)^{-1} & * \\
R^{\frac{1}{2}} K(\alpha) C_{Z} G(\alpha) & 0 & -I
\end{array}\right)<0
$$

where

$$
\Pi_{11}=-G(\alpha)^{T}-G(\alpha)+P(\theta)^{-1}+G(\alpha)^{T} Q_{\hat{x}} G(\alpha),
$$

$Q_{\hat{x}}$ and $R$ are the weighting matrices in performance index (28), then the static output feedback controller (33) is a guaranteed cost controller and the performance index satisfies $\widehat{J} \leq \widehat{x}_{0}^{T} P \widehat{x}_{0}$, where $\widehat{x}_{0}$ is the initial state of augmented error system (27).

Proof: Based on (27) and (33), the following equation will be obtained:

$$
\widehat{x}(k+1)=\left[\widehat{A}(\theta)+\widehat{B}(\theta) K(\alpha) C_{Z}\right] \widehat{x}(k) .
$$

Define a Lyapunov functional as

$$
V(\widehat{x}(k))=\widehat{x}(k)^{T} P(\theta) \widehat{x}(k),
$$

where $P(\theta)$ is a positive definitive matrix. The difference of Lyapunov function (36) can be obtained as

$$
\begin{aligned}
\Delta V(\widehat{x}(k))= & V(\widehat{x}(k+1))-V(\widehat{x}(k)) \\
= & \widehat{x}(k+1)^{T} P(\theta) \widehat{x}(k+1)-\widehat{x}(k)^{T} P(\theta) \widehat{x}(k) \\
= & \widehat{x}(k)^{T}\left[\widehat{A}(\theta)+\widehat{B}(\theta) K(\alpha) C_{Z}\right]^{T} P(\theta)[\widehat{A}(\theta)+ \\
& \left.\widehat{B}(\theta) K(\alpha) C_{Z}\right] \widehat{x}(k)-\widehat{x}(k)^{T} P(\theta) \widehat{x}(k) .
\end{aligned}
$$


If the following condition

$$
\begin{aligned}
& {\left[\widehat{A}(\theta)+\widehat{B}(\theta) K(\alpha) C_{Z}\right]^{T} P(\theta)\left[\widehat{A}(\theta)+\widehat{B}(\theta) K(\alpha) C_{Z}\right]} \\
& -P(\theta)+Q_{\hat{x}}+C_{Z}^{T} K(\alpha)^{T} R K(\alpha) C_{Z}<0
\end{aligned}
$$

holds, then it follows from (37), one has

$$
\Delta V(\widehat{x}(k))<-\widehat{x}(k)^{T}\left(Q_{\hat{x}}+C_{Z}^{T} K(\alpha)^{T} R K(\alpha) C_{Z}\right) \widehat{x}(k)<0 .
$$

Thus, according to Lyapunov's stability theory, the closed-loop system (35) is robust asymptotically stable. Furthermore, from (39), we have

$$
\widehat{x}(k)^{T}\left(Q_{\hat{x}}+C_{Z}^{T} K(\alpha)^{T} R K(\alpha) C_{Z}\right) \widehat{x}(k)<-\Delta V(\widehat{x}(k)) .
$$

Summing both sides of the above inequality from $k=1$ to $k=\infty$, it yields

$$
\bar{J}=\sum_{k=1}^{\infty}\left[\widehat{x}(k)^{T} Q_{\hat{x}} \widehat{x}(k)+\Delta u(k)^{T} R \Delta u(k)\right] \leq \widehat{x}_{0}^{T} P \widehat{x}_{0} .
$$

On the other hand, applying the Schur complement formula, condition (38) can be equivalent to

$$
\left(\begin{array}{ccc}
-P(\theta)+Q_{\hat{x}} & * & * \\
\widehat{A}(\theta)+\widehat{B}(\theta) K(\alpha) C_{Z} & -P(\theta)^{-1} & * \\
R^{\frac{1}{2}} K(\alpha) C_{Z} & 0 & -I
\end{array}\right)<0 .
$$

Pre- and post-multiplying inequality (42) by diag $\left\{G(\alpha)^{T}, I, I\right\}$ and its transpose, respectively, one gets

$$
\left(\begin{array}{ccc}
\Lambda_{11} & * & * \\
{\left[\widehat{A}(\theta)+\widehat{B}(\theta) K(\alpha) C_{Z}\right] G(\alpha)} & -P(\theta)^{-1} & * \\
R^{\frac{1}{2}} K(\alpha) C_{Z} G(\alpha) & 0 & -I
\end{array}\right)<0
$$

where $\Lambda_{11}=-G(\alpha)^{T} P(\theta) G(\alpha)+G(\alpha)^{T} Q_{\hat{x}} G(\alpha)$.

From Lemma 2.6, it follows that :

$$
-G(\alpha)^{T} P(\theta) G(\alpha) \leq-G(\alpha)^{T}-G(\alpha)+P(\theta)^{-1} .
$$

Therefore, based on (43) and (44), (43) is guaranteed by (34). The proof is completed.

The following result is equivalent to Theorem 4.1. 
Theorem 4.2. For the augmented system (27) with the performance index (28), if there exist positive defined parameter-dependent matrix $X(\theta)$, reversible matrices $W, U, G(\alpha)$ and a scalar $\beta$ such that

$$
\Psi(\theta, \alpha)=\left(\begin{array}{cccc}
\Psi_{11} & * & * & * \\
\Psi_{21} & -X(\theta) & * & * \\
R^{\frac{1}{2}} L(\alpha) Q_{\hat{x}} & 0 & -I & * \\
C_{z} G(\alpha)-U Q_{\hat{x}} & \Psi_{42} & \beta W^{T} L(\alpha)^{T} R^{\frac{1}{2}} & \Psi_{44}
\end{array}\right)<0
$$

where

$$
\begin{aligned}
\Psi_{11} & =-G(\alpha)-G(\alpha)^{T}+X(\theta)+G(\alpha)^{T} Q_{\hat{x}} G(\alpha), \\
\Psi_{21} & =\widehat{A}(\theta) G(\alpha)+\widehat{B}(\theta) L(\alpha) Q_{\hat{x}}, \\
\Psi_{42} & =\beta W^{T} L(\alpha)^{T} \widehat{B}(\theta)^{T}, \\
\Psi_{44} & =-\beta U M-\beta U^{T} M^{T},
\end{aligned}
$$

$Q_{\hat{x}}$ and $R$ are the weighting matrices in performance index (28), then the conclusion of Theorem 4.1 holds.

Proof: Denote

$$
\widetilde{A}=\left(\begin{array}{c}
\widehat{A} \\
0
\end{array}\right), \quad \widetilde{B}=\left(\begin{array}{c}
\widehat{B} \\
R^{\frac{1}{2}}
\end{array}\right), \quad \Phi(\theta)=\left(\begin{array}{cc}
X(\theta) & 0 \\
0 & I
\end{array}\right) .
$$

Inequality (45) can be written as

$$
\left(\begin{array}{ccc}
\Psi_{11} & * & * \\
\widetilde{A}(\theta) G(\alpha)+\widetilde{B}(\theta) L(\alpha) Q_{\hat{x}} & -\Phi(\theta) & * \\
C_{Z} G(\alpha)-U Q_{\hat{x}} & \beta W^{T} L(\alpha)^{T} \widetilde{B}(\theta)^{T} & \Psi_{44}
\end{array}\right)<0
$$

or

$$
\left(\begin{array}{cc}
\left(\begin{array}{cc}
\Psi_{11} & * \\
\widetilde{A}(\theta) G(\alpha)+\widetilde{B}(\theta) L(\alpha) Q_{\hat{x}} & -\Phi(\theta)
\end{array}\right) & * \\
\Xi_{31} & \\
\Psi_{44}
\end{array}\right)<0
$$

where

$$
\Xi_{31}=\beta W^{T} L(\alpha)^{T} \widetilde{B}(\theta)^{T}\left[\begin{array}{ll}
0 & I
\end{array}\right]+U W W^{-1} U^{-1}\left(C_{Z} G(\alpha)-U Q_{\hat{x}}\right)\left[\begin{array}{ll}
I & 0
\end{array}\right] .
$$


We further denote

$$
\begin{aligned}
& T=\left[\begin{array}{cc}
-G(\alpha)-G(\alpha)^{T}+X(\theta)+G(\alpha)^{T} Q_{\hat{x}} G(\alpha) & * \\
\widetilde{A}(\theta) G(\alpha)+\widetilde{B}(\theta) L(\alpha) Q_{\hat{x}} & -\Phi(\theta)
\end{array}\right], \\
& N=U M, \quad R=\left[\begin{array}{c}
0 \\
I
\end{array}\right] \widetilde{B}(\theta) L(\alpha) W, \\
& S=W^{-1} U^{-1}\left(C_{Z} G(\alpha)-U Q_{\hat{x}}\right)\left[\begin{array}{ll}
I & 0
\end{array}\right] .
\end{aligned}
$$

By Lemma 2.5, inequality (48) can guarantee that

$$
\begin{aligned}
& \left(\begin{array}{cc}
-G(\alpha)-G(\alpha)^{T}+X(\theta)+G(\alpha)^{T} Q_{\hat{x}} G(\alpha) & * \\
\widetilde{A}(\theta) G(\alpha)+\widetilde{B}(\theta) L(\alpha) Q_{\hat{x}} & -\Phi(\theta)
\end{array}\right) \\
+ & \operatorname{sym}\left(\left[\begin{array}{c}
0 \\
I
\end{array}\right] \widetilde{B}(\theta) L(\alpha) W W^{-1} U^{-1}\left(C_{Z} G(\alpha)-U Q_{\hat{x}}\right)\left[\begin{array}{ll}
I & 0
\end{array}\right]\right) \\
= & \left(\begin{array}{cc}
-G(\alpha)-G(\alpha)^{T}+X(\theta)+G(\alpha)^{T} Q_{\hat{x}} G(\alpha) & * \\
\widetilde{A}(\theta) G(\alpha)+\widetilde{B}(\theta) L(\alpha) Q_{\hat{x}} & -\Phi(\theta)
\end{array}\right) \\
+ & \operatorname{sym}\left(\left[\begin{array}{c}
0 \\
I
\end{array}\right] \widetilde{B}(\theta) L(\alpha) U^{-1}\left(C_{Z} G(\alpha)-U Q_{\hat{x}}\right)\left[\begin{array}{cc}
I & 0
\end{array}\right]\right) \\
= & \left(\begin{array}{cc}
-G(\alpha)-G(\alpha)^{T}+X(\theta)+G(\alpha)^{T} Q_{\hat{x}} G(\alpha) & * \\
\widetilde{A}(\theta) G(\alpha) & -\Phi(\theta)
\end{array}\right) \\
+ & \operatorname{sym}\left(\left[\begin{array}{c}
0 \\
I
\end{array}\right] \widetilde{B}(\theta) L(\alpha) U^{-1}\left(C_{Z} G(\alpha)+U Q_{\hat{x}}-U Q_{\hat{x}}\right)\left[\begin{array}{ll}
I & 0
\end{array}\right]\right) \\
< & 0 .
\end{aligned}
$$

Setting $K(\alpha)=L(\alpha) U^{-1}$, then inequality (49) becomes

$$
\begin{aligned}
& \left(\begin{array}{cc}
-G(\alpha)-G(\alpha)^{T}+X(\theta)+G(\alpha)^{T} Q_{\hat{x}} G(\alpha) & * \\
\widetilde{A}(\theta) G(\alpha) & -\Phi(\theta)
\end{array}\right) \\
& +\operatorname{sym}\left(\left[\begin{array}{c}
0 \\
I
\end{array}\right] \widetilde{B}(\theta) K(\alpha)\left(C_{Z} G(\alpha)\right)\left[\begin{array}{ll}
I & 0
\end{array}\right]\right) \\
& =\left(\begin{array}{cc}
-G(\alpha)-G(\alpha)^{T}+X(\theta)+G(\alpha)^{T} Q_{\hat{x}} G(\alpha) & * \\
\widetilde{A}(\theta) G(\alpha)+\widetilde{B}(\theta) K(\alpha) C_{Z} G(\alpha) & -\Phi(\theta)
\end{array}\right)<0 .
\end{aligned}
$$


By some calculation, it can be found that

$$
\begin{aligned}
& \left(\begin{array}{cc}
-G(\alpha)-G(\alpha)^{T}+X(\theta)+G(\alpha)^{T} Q_{\hat{x}} G(\alpha) & * \\
\widetilde{A}(\theta) G(\alpha)+\widetilde{B}(\theta) K(\alpha) C_{Z} G(\alpha) & -\Phi(\theta)
\end{array}\right) \\
& =\left(\begin{array}{ccc}
-G(\alpha)-G(\alpha)^{T}+X(\theta)+G(\alpha)^{T} Q_{\hat{x}} G(\alpha) & * & * \\
\widehat{A}(\theta) G(\alpha)+\widehat{B}(\theta) K(\alpha) C_{Z} G(\alpha) & -X(\theta) & * \\
R^{\frac{1}{2}} K(\alpha) C_{Z} G(\alpha) & 0 & -I
\end{array}\right) .
\end{aligned}
$$

Setting $X(\theta)=P(\theta)^{-1}$, it follows from Theorem 4.1, Theorem 4.2 holds.

It should be pointed out that the condition (45) in Theorem 4.2 is not a convex problem. The following theorem can translate it into an LMI problem.

Theorem 4.3. Given a scalar $\beta$ and matrix $W$, if there exist $X_{i}>0, U$, $L_{i}, G_{i}(i=1,2,3 \ldots, s)$ with appropriate dimensions such that

$$
\Pi_{i j}<0(i, j \in 1,2, \cdots, s)
$$

where

$$
\Pi_{i j}=\left(\begin{array}{ccccc}
-G_{j}-G_{j}^{T}+X_{i} & * & * & * & * \\
\widehat{A}_{i} G_{j}+\widehat{B}_{i} L_{j} Q_{\hat{x}} & -X_{i} & * & * & * \\
R^{\frac{1}{2}} L_{j} Q_{\hat{x}} & 0 & -I & * & * \\
C_{z i} G_{j}-U Q_{\hat{x}} & \beta W^{T} L_{j}^{T} \widehat{B}_{i}^{T} & \beta W^{T} L_{j}^{T} R^{\frac{1}{2}} & \Psi_{44} & * \\
Q_{\hat{x}}^{\frac{1}{2}} G_{j} & 0 & 0 & 0 & -I
\end{array}\right),
$$

$Q_{\hat{x}}$ and $R$ are the weighting matrices in performance index (28), then the augmented system (27) is robustly asymptotically stable under the guaranteed cost controller (32), and the gain matrix can be obtained by $K_{i}=L_{i} U^{-1}$. Moreover, the corresponding closed-loop performance index satisfies $\widehat{J} \leq$ $\sum_{i=1}^{s} \widehat{x}_{0}^{T} X_{i}^{-1} \widehat{x}_{0}$.

Proof: From (23) and take into account the definition of $K(\alpha), G(\alpha)$, one gets

$$
\Pi(\theta, \alpha)=\sum_{i=1}^{s} \sum_{j=1}^{s} \theta_{i} \alpha_{j} \Pi_{i j} .
$$

In fact, inequality $(52)$ implies $\Pi(\theta, \alpha)<0$. As a result, the condition of Theorem 4.2 holds. The proof is completed.

Based on Theorem 4.3, the following conclusion can be obtained easily. 
Corollary 4.4. Given a scalar $\beta$ and matrix $W$, if there exist $X>0, U, L$ and $G$ with appropriate dimensions such that

$$
\Psi_{i}<0(i \in 1,2,3, \ldots, s),
$$

where

$$
\Psi_{i}=\left(\begin{array}{ccccc}
-G-G^{T}+X & * & * & * & * \\
\widehat{A}_{i} G+\widehat{B}_{i} L Q_{\hat{x}} & -X & * & * & * \\
R^{\frac{1}{2}} L Q_{\hat{x}} & 0 & -I & * & * \\
C_{z i} G-U Q_{\hat{x}} & \beta W^{T} L^{T} \widehat{B}_{i}^{T} & \beta W^{T} L^{T} R^{\frac{1}{2}} & \Psi_{44} & * \\
Q_{\hat{x}}^{\frac{1}{2}} G & 0 & 0 & 0 & -I
\end{array}\right)<0,
$$

then the augmented system (27) is robustly asymptotically stable under the guaranteed cost controller

$$
\Delta u(k)=K Z(k)=L U^{-1} Z(k) .
$$

The gain matrix can be obtained by $K=L U^{-1}$. Moreover, the corresponding closed-loop performance index satisfies $\widehat{J} \leq \widehat{x}_{0}^{T} X^{-1} \widehat{x}_{0}$.

\section{Numerical example}

Consider the discrete-time system (1) with

$$
\begin{aligned}
& A(\theta)=\left(\begin{array}{ll}
0.60 & 0.10 \\
0.45 & 0.80
\end{array}\right) \theta_{1}+\left(\begin{array}{cc}
0.95 & 0 \\
0.10 & 0.96
\end{array}\right) \theta_{2}, \\
& B(\theta)=\left(\begin{array}{l}
0.35 \\
0.14
\end{array}\right) \theta_{1}+\left(\begin{array}{l}
0.30 \\
0.34
\end{array}\right) \theta_{2}, \quad E=\left(\begin{array}{l}
0.02 \\
0.05
\end{array}\right), C=\left[\begin{array}{ll}
0.9 & 0.5
\end{array}\right] .
\end{aligned}
$$

Assume that the reference signal is

$$
r(k)=\sin \left(\frac{2 \pi}{10} k\right)+0.25 \sin \left(\frac{4 \pi}{10} k\right)+0.5 \sin \left(\frac{6 \pi}{10} k\right)
$$

and the disturbance signal is $d(k)=\sin \left(\frac{2 \pi}{10} k\right)$.

Take the uncertain parameters $\theta_{1}=0.20, \theta_{2}=0.80$, the adjustable parameters $\beta=0.40$ and $\alpha_{1}=0.80, \alpha_{2}=0.20, Q=2 C_{Z}, R=1, W=$ $0.3 C_{Z} C_{Z}^{T}$.

The simulation results are presented for the following three situations, namely, (i) $M_{r}=7, M_{d}=6$, (ii) $M_{r}=5, M_{d}=4$ and (iii) $M_{r}=2, M_{d}=1$. By 
Figure 3: The reference signal and the output of system

solving LMIs (52) in Theorem 4.3, the feedback gain matrix $K_{i}=L_{i} U^{-1}(i=$ $1,2)$ can be derived, then the feedback gain $K=\alpha_{1} K_{1}+\alpha_{2} K_{2}$ is obtained.

When $M_{r}=7, M_{d}=6$, the following parameters are obtained:

$$
\begin{aligned}
& K_{e}=\left[\begin{array}{llllll}
0.0279 & -0.0065 & -0.0013 & -0.0004 & -0.0003 & -0.0007
\end{array}\right. \\
& \begin{array}{llll}
-0.0011 & 0.0001 & 0.0376 & 2.3171
\end{array}, \quad K_{y}=\left[\begin{array}{l}
-2.2477
\end{array}\right], \\
& K_{r}=\left[\begin{array}{llll}
-0.0037 & 2.3171 & 0.0377 & -0.0001
\end{array}\right. \\
& -0.0014-0.0010-0.0007-0.0009] \\
& K_{d}=\left[\begin{array}{lllllll}
-0.1006 & -0.0005 & 0.0006 & 0.0003 & 0.0002 & 0.0001 & 0.0001
\end{array}\right] .
\end{aligned}
$$

When $M_{r}=5, M_{d}=4, K_{e}, K_{y}, K_{r}$ and $K_{d}$ will be:

$$
\begin{aligned}
K_{e}= & {\left[\begin{array}{llllll}
0.0212 & -0.0064 & -0.0013 & -0.0004 & -0.0003 & -0.0007 \\
& -0.0011 & 0.0002 & 0.0376 & 2.3167
\end{array}\right], \quad K_{y}=\left[\begin{array}{llll}
-2.2477
\end{array}\right] } \\
K_{r}= & {\left[\begin{array}{llllll}
-0.0029 & 2.3160 & 0.0372 & -0.0004 & -0.0018 & -0.0015
\end{array}\right] } \\
K_{d}= & {\left[\begin{array}{llllll}
-0.1005 & -0.0004 & 0.0006 & 0.0004 & 0.0002
\end{array}\right] }
\end{aligned}
$$

When $M_{r}=2, M_{d}=1$, the following will be obtained:

$$
\begin{aligned}
K_{e} & =\left[\begin{array}{llllll}
0.0445 & -0.0046 & -0.0018 & -0.0008 & -0.0008 & -0.0013 \\
& -0.0018 & -0.0005 & 0.0397 & 2.3239
\end{array}\right], \quad K_{y}=-2.2532 \\
K_{r} & =\left[\begin{array}{lllll}
-0.0008 & 2.3233 & 0.0386
\end{array}\right], \quad K_{d}=\left[\begin{array}{llll}
-0.1005 & -0.0002
\end{array}\right]
\end{aligned}
$$

Set the initial value of $x(k)$ be $x(0)=\left[\begin{array}{ll}0 & 0\end{array}\right]^{T}$. Fig. 3 shows the reference signal $r(k)$ and the outputs $y(k)$ of system (1). The tracking errors and 
Figure 4: The tracking errors

Figure 5: The control inputs

control input are depicted in Fig. 4 and Fig. 5, respectively. It can be seen from Fig. 3 and Fig. 4, the outputs can all track the reference signal accurately. Particularly, the repetitive control with preview compensator can effectively reduce the tracking error. In addition, the tracking error peak decrease of the control system is faster with the increase of the preview length.

For comparison, we let $M_{r}=0, M_{d}=0$ to design a conventional guaranteed-cost repetitive-controller [30,31]. The best results are shown in Fig. 6 and Fig. 7. From the comparison, we can see that the preview repetitive controller provides better performance than the repetitive controller with no preview compensation does. 
Figure 6: The reference signal and the output of system with no preview.

Figure 7: The tracking error with no preview.

\section{Conclusion}

This paper presented a design method of guaranteed cost preview repetitive controller for a class of polytopic uncertain discrete-time systems. Using the $L$-order forward difference operator, an augmented dynamic system was first constructed. Based on that, a guaranteed cost static output feedback controller was then designed. By incorporating this controller into the original system, the guaranteed-cost preview repetitive controller was obtained. Simulation results verified the proposed method to be very effective.

[1] T. Katayama, T. Ohki, T. Inoue and T. Kato, Design of an optimal controller for a discrete-time system subject to previewable demand, International Journal of Control, Vol. 23, no. 3, pp. 667-699, 1985. 
[2] T. Tsuchiya, E. Tadashi, Translate by F. C. Liao, Digital preview and predictive control. Beijing: Beijing Science and Technology Press, 1994.

[3] F. Liao, T. Tsuchiya, T. Egami, and Y. Xin, Unified approach to optimal preview servo systems and optimal preview for compensated systems, Chinese Journal of Automation, Vol. 10, no. 4, pp. 329-336, 1998.

[4] T. Kiyotsugu, Robust servomechanism with preview action for polytopic uncertain systems, International Journal of Robust And Nonlinear Control, Vol. 10, no. 2, pp. 101-111, 2000.

[5] A. Kojima and S. Ishijima, $H_{\infty}$ preview tracking in output feedback setting, International Journal of Robust And Nonlinear Control, Vol. 14, no. 7, pp. 627-641, 2004.

[6] D. R. Kenneth, N. C. Martins, Optimal preview control of Markovian jump linear systems, IEEE Transactions on Automatic Control, Vol. 54, no. 9, pp. 2260-2266, 2009.

[7] R. S. Prabakar, C. Sujatha, S. Narayanan, Optimal semi-active preview control response of a half car vehicle model with magnetorheological damper, Journal of Sound and Vibration, Vol. 326, no. 5, pp. 400-420, 2009.

[8] L. Wang, J. Zheng, M. Fu, Optimal preview control of a dual-stage actuator system for triangular reference tracking, IEEE Transactions on Control Systems Technology, Vol. 22, no. 6, pp. 2408-2416, 2014.

[9] A. T. Salton, Z. Chen, J. Zheng, M. Fu, Constrained optimal preview control of dual-stage actuators, IEEE/ASME Transactions on Mechatronics, Vol. 21, no. 2, pp. 1179-1184, 2016.

[10] J. N. Strohm, B. Lohmann, Optimal feedforward preview control by fir filters, IFAC-PapersOnLine, Vol. 50, no. 1, pp. 5115-5120, 2017.

[11] M. G. Jadidi, E. Hashemi, Optimal preview control of the nao biped robot using a ukf-based state observer, 2016 IEEE International Conference on Advanced Intelligent Mechatronics, Banff, Alberta, Canada, July 12-15, 2016. 
[12] N. Birla, A. Swarup, Optimal preview control: A review, Optim. Control Appl. Meth., Vol. 36, no. 1, pp. 241-268, 2015.

[13] K. Takaba, Robust preview tracking control for polytopic uncertain systems, Proceedings of the 37th IEEE Conference on Decision and Control, Tampa, Florida, USA, Dece 23-25, 1998.

[14] F. Liao, J. L. Wan and G. H. Yang, LMI-based reliable robust preview tracking control against actuator faults, Proceedings of the American Control Conference, Arlington, VA June 25-27, 2001.

[15] D. Wang, F. Liao, M. Tomizuka, Adaptive preview control for piecewise discrete-time systems using multiple models, Applied Mathematical Modelling, Vol. 40, no. 1, pp. 9932-9946, 2016.

[16] Z. Y. Zhen, Research development in preview control theory and applications, Acta Autom. Sin.,, Vol. 42, no. 2, pp. 172-188, 2016.

[17] L. Li, F. C. Liao, Parameter-dependent preview control with robust tracking performance, IET Control Theory Appl., Vol. 11, no. 1, pp. 38-46, 2017.

[18] F. Liao, L. Li, Robust preview control for uncertain discrete-time systems based on LMI, Optim Control Appl Meth., doi: 10.1002/oca.2308, 2017.

[19] T. Inoue, M. nakano, S. Iwai, High accuracy control of a proton synchrotron magnet power supply, In Proceedings of the 8th Internat Fedaration on Automatic Control, Kyoto, Japan, pp. 216C221, 1981.

[20] Y. Wang, R. Wang, X. Xie, H. Zhang, Observer-based $H_{\infty}$ fuzzy control for modified repetitive control systems, Neurocomputing, Vol. 286, no. 19, pp. 141-149, 2018.

[21] R. D. Hanson, T. C. Tsao, Discrete-time repetitive control of LTI systems sampled at a periodic rate, In IFAC Proceedings, Vol. 29, no. 1, pp. 1458-1463, 1996.

[22] J. H. She, Y. Pan and M. Nakano, Repetitive control system with variable structure controller, The 6th International Workshop on Variable Structure Systems, Gold Coast, Australia, December 2000. 
[23] M. Sun, Y. Wang, D. Wang, Variable-structure repetitive control: a discrete-time strategy, IEEE Transactions on Industrial Electronics, Vol. 52, no.2, pp. 610-616, 2005.

[24] J. Fan, T. Kobayashi, Discrete repetitive control via positive real compensation, Transactions of the Society of Instrument and Control Engineers, Vol. 33, no.4, pp. 308-310, 2009.

[25] S. G. Yuan, M. Wu, B. G. Xu, R. J. Liu, Design of discrete-time repetitive control system based on two-dimensionalmodel, International Journal of Automation and Computing, Vol. 9, no.2, pp. 165-168, 2012.

[26] L. Zhou, J. H. She, M. Wu, Design of a design of a discrete-time outputfeedback based repetitive-control system, International Journal of Automation and Computing, Vol. 10, no.4, pp. 343-349, 2013.

[27] Z. H. Wang, L. Z. Yi, Y. H. Lan, C. X. Chen, Design of observer-based discrete repetitive-control system based on 2D model, J. Cent. South. Univ., Vol. 21, no. 1, pp. 4236-42436, 2014.

[28] J. H. Park, Guaranteed cost stabilization of neutral differential systems with parametric uncertainty, Int. Journal of Computational and Applied Mathematics, Vol. 151, No. 15, pp. 371-382, 2003.

[29] P. Shi, K. K. Boukas, Y. Shi and R. K. Agarwal, Optimal guaranteed cost control of uncertain discrete time-delay systems, Int. Journal of Computational and Applied Mathematics, Vol. 157, No. 15, pp. 435-451, 2004 .

[30] C. Fu, Y. He, M. Wu, S. Yuan, Guaranteed cost robust discrete repetitive control based on two-dimension model, Proceedings of the 29th Chinese Control Conference, Beijing, China, July 29-31, 2010.

[31] M. Wu Y. H. Lan, J. H. She, Y. He, Design of nonfragile guaranteedcost repetitive-control system based on two-dimensional model, Asian Journal of Control, Vol. 14, No. 1, pp. 109-124, 2012.

[32] K. Takaba, Robust preview tracking control for polytopic uncertain systems, Proceedings of the 37th IEEE Conference on Decision and Control, Tampa, Florida, USA, December 1998. 
[33] L. Li, F. C. Liao, Design of a preview controller for discrete-time systems based on LMI, Math. Prob. Eng., Vol. 15, 1-12, 2015.

[34] M. Tomizuka, T. C. Tsao, K. K. Chew, Analysis and synthesis of discrete-time repetitive controllers, Irans. ASME, J. Dynam. Syset., Measu., Contr., Vol. 111, no. 353, pp. 353-358, 1989.

[35] X. H. Chang, L. Zhang, J. H. Park, Robust static output feedback $H_{\infty}$ control for uncertain fuzzy systems, Fuzzy Sets and Systems, Vol. 273, pp. 87-104, 2015.

[36] A. Nasiri, S. K. Nguang, A. Swain, et al, Robust output feedback controller design of discrete-time TakagiCSugeno fuzzy systems: a nonmonotonic Lyapunov approach, IET Control Theory Appl., Vol. 10, no. 5, pp. 545-553, 2016. 